\title{
Operational Experience: Integration of ASPR Data into ESSENCE-FL during the RNC
}

\author{
Carrie Eggers*1, Dina Passman², Aaron Chern¹, Dara Spector ${ }^{2}$, Aaron Kite-Powell ${ }^{1}$, Tim \\ Davis $^{2}$, Wayne Loschen ${ }^{3}$, Joe Lombardo ${ }^{3}$, Douglas Char ${ }^{2}$ and Janet Hamilton ${ }^{1}$
}

${ }^{1}$ Florida Department of Health, Bureau of Epidemiology, Tallahassee, FL, USA; ${ }^{2}$ Health and Human Services, Office of the Assistant Secretary for Preparedness and Response, Washington, D.C., DC, USA; ${ }^{3}$ Johns Hopkins University Applied Physics Laboratory, Laurel, MD, USA

\section{Objective}

The Florida Department of Health (FDOH), Bureau of Epidemiology, partnered with the U.S. Department of Health and Human Services (HHS) Office of the Assistant Secretary for Preparedness and Response (ASPR) to improve surveillance methods in post disaster or response events. A new process was implemented for conducting surveillance to monitor injury and illness for those presenting for care to ASPR assets such as Disaster Medical Assistance Team (DMAT) sites when they are operational in the state. The purpose of the current work was to field test and document the operational experience of the newly implemented ASPR data module in ESSENCEFL (syndromic surveillance system) to receive near real-time automated data feeds when ASPR federal assets were deployed in Florida during the 2012 Republican National Convention (RNC).

\section{Introduction}

Florida has implemented various surveillance methods to augment existing sources of surveillance data and enhance decision making with timely evidence based assessments to guide response efforts post-hurricanes. Historically, data collected from deployed federal assets have been an integral part of this effort. However, a number of factors have made this type of surveillance challenging: logistical issues of field work in a post-disaster environment, the resource intensive manual data collection process from DMAT sites, and delayed analysis and interpretation of these data to inform decision makers. The ESSENCE-FL system is an automated and secure web-based application accessed by FDOH epidemiologists and staff at participating hospitals.

\section{Methods}

ESSENCE-FL was configured by the Johns Hopkins University Applied Physics Laboratory (JHU/APL) to receive ASPR electronic medical record (EMR) data. A scheduled program to generate data files for FDOH was created using SAS Enterprise Business Intelligence (EBI) software and a script was set up on the ASPR server to send an updated file via secure file transfer protocol (sftp) every 15 minutes. A case definition was created by ASPR field teams to identify which encounter visits would be entered into the electronic medical record (EMR) and received in ESSENCE-FL. To assess completeness of data elements and total patient encounters received in ESSENCE-FL, DMAT field teams maintained Excel line lists of patient encounters and emailed them to FDOH three times daily during the RNC. ASPR data were reviewed and analyzed by FDOH staff multiple times a day in near real time utilizing the existing ESSENCE-FL robust analysis tools.

\section{Results}

Three separate ASPR missions were deployed to Florida to support the RNC. ASPR EMR data files were received at 15-minute intervals by ESSENCE-FL from the ASPR central server during each day of the 2012 RNC (August 26-31). Reduced patient counts within ESSENCE-FL as compared with DMAT-maintained Excel line lists indicated an incomplete input, upload, or transfer of patient data from one of two ASPR sites to the central ASPR servers. Although only 11 of 34 total patient encounters were received by ESSENCE-FL during the event, the system design enabled users to run specific queries and display the results of their queries in time series graphs, pie and bar charts, GIS maps, dashboards, and statistical tables.

\section{Conclusions}

There is a great need to have timely access to data sources to enhance disease surveillance efforts and help guide decision makers' situational awareness and disease control efforts during a response. The FDOH, Bureau of Epidemiology's collaboration with JHU/APL and ASPR takes advantage of ASPR's EMR-S to make data sharing and analysis efficient as evidenced during the RNC. Automated data feeds to ESSENCE-FL removed resource intensive manual data collection by public health, improved standardization of syndrome and demographic categorizations, increased access to these data by local, state, and federal epidemiologists in a timely manner, and expedited analysis and interpretation for situational awareness. Future recommendations include pre-event testing of the entire data flow process, establishing an on-site specialist to immediately assist with any issues, greater understanding of the field team use of the EMR-S, and ensuring field staff is aware of data quality needs for effective public health surveillance.

\section{Keywords}

surveillance; response; disaster

\section{${ }^{*}$ Carrie Eggers}

E-mail: Carrie_Eggers@doh.state.fl.us 